Poster (M16)

Preinvasive Disease of Cervix, Vulva, and Vagina

https://doi.org/10.3802/jgo.2021.32.S1.M16

\section{Efficacy of lidocaine spray for pain reduction during colposcopy-directed cervical biopsy: a randomized controlled trial}

\section{Charuwan Saeteng," Ornwitsanate Mongkolmafai, Kittipat Charoenkwan, Prapaporn Suprasert, Jatupol Srisomboon}

Chiang Mai University, Chiang Mai, Thailand (nokctantipa@gmail.com)

Objective: To evaluate the efficacy of lidocaine spray in reducing pain during colposcopy-directed cervical biopsy (CDB).

Methods: From December 2017 through February 2019, 312 women undergoing $\mathrm{CDB}$ were enrolled. The participants were randomized to 3 groups: group 1 (lidocaine spray), lidocaine spray was applied thoroughly to the cervix; group 2 (placebo), normal saline was applied thoroughly to the cervix; group 3 (control), no anesthetic agent was applied to the cervix. Each woman completed a $10-\mathrm{cm}$ visual analog scale for subjective pain experience at three-time points: baseline, immediately after biopsy, and 10 minutes after the procedure. The primary outcome of this study was the biopsy pain score.

Results: Of the 312 women, 104 were randomly assigned to each study group. The clinic-pathological and procedure-related characteristics of the participants in all groups were similar. The baseline, the biopsy, and the post-procedure pain scores were comparable among the three groups. We found a significant difference in the pain scores changes from baseline to biopsy and from baseline to postprocedure among the three groups. However, when compared to the lidocaine spray group directly to the control group, there was no statistically significant difference in the pain scores changes from baseline to biopsy $(p=0.06)$ and from baseline to postprocedure $(p=0.29)$. There was no complication with the intervention observed.

Conclusion: The application of lidocaine spray to the cervix has a small benefit in reducing pain associated with CDB. However, the intervention is safe and may be considered in nulliparous and/or overly anxious women undergoing the procedure.

Poster (M17)

Preinvasive Disease of Cervix, Vulva, and Vagina

https://doi.org/10.3802/jgo.2021.32.S1.M17

\section{Treatment outcomes of patients with cervical intraepithelial neoplasia or invasive carcinoma who underwent loop electrosurgical excision procedure (LEEP)}

Jakkapan Khunnarong, ${ }^{1, *}$ Nitinan Bunyasontikul, ${ }^{2}$ Siriwan Tangjitgamol ${ }^{3}$

'Faculty of Medicine Vajira Hospital, Navamindradhiraj University, Bangkok, Thailand (jak020@hotmail.com)

${ }^{2}$ Obstetrics and Gynecology Section, Somdejprapinklao Hospital, Bangkok, Thailand

${ }^{3}$ Women's Health Center, MedPark Hospital, Bangkok, Thailand

Objective: To study treatment outcomes of cervical intraepithelial neoplasia (CIN) or cancer patients who underwent loop electrosurgical excision procedure (LEEP) in terms of primary outcome and factors associated with persistent/recurrence. Methods: Patients with CIN or cancer who underwent LEEP from January 2007 to December 2015 were reviewed. Data collected were age, parity, menopausal status, human immunodeficiency virus (HIV) infection, smoking, cervical cytology, histopathology from cervical biopsy and LEEP including margin status, final histopathology, and follow-up data.

Results: The mean age of 385 patients was $41.9+10.8$ years (range, 18-79 years). Majority were multiparous (81.6\%) and premenopausal $(78.2 \%)$. There were $15.3 \%$ of patients had HIV infection. The most common cervical cytology was HSIL (44.1\%) followed by ACS-US (21\%). Minor complications of bleeding or infection from LEEP were encountered $7.3 \%$. Among 153 patients (39.7\%) who had positive margin(s), 43 underwent second LEEP whereas 76 had hysterectomy. From all patients, 47 had failure aftertreatment (12.2\%): being either persistence (30 patients; $7.8 \%$ ) or recurrence (17 patients; $4.4 \%$ ). Factors associated with persistence or recurrence by multivariate analysis were age $\geq 55$ years old, HIV infection, final diagnosis of invasive cancer, and positive of endocervical margin or both ecto- and endo-cervical margins.

Conclusion: LEEP had low rate of persistence/recurrence. Age $\geq 55$ years old, HIV infection, final diagnosis of cancer, and positive of endocervical or both endo- and ecto-surgical margin(s) were significantly associated with persistent or recurrent diseases.

Poster (M18)

Quality of Life, Nursing \& Supportive Care https://doi.org/10.3802/jgo.2021.32.S1.M18

\section{Quality of life and psychological aspects among ovarian cancer survivors compare to normal female population at Bhumibol Adulyadej Hospital}

\author{
Wanlaya Onwatanasrikul,," Worrawan Sirichai, \\ Komsan Suwanalerk ${ }^{2}$ \\ 'Bhumibol Adulyadej Hospital, Bangkok, Thailand \\ (wanlaya.onw@gmail.com) \\ ${ }^{2}$ Thammasat University Hospital, Pathum Thani, Thailand
}

